EPJ Web of Conferences 31, 00033 (2012)

DOI: $10.1051 /$ epjconf/20123100033

(C) Owned by the authors, published by EDP Sciences - SIF, 2012

\title{
Particle-particle correlations: Femtoscopy and tools for spectroscopy
}

\author{
G. VERDE
}

\author{
Istituto Nazionale di Fisica Nucleare, Sezione di Catania \\ I-95123 Catania, Italy
}

\begin{abstract}
A review of particle-particle correlation studies at intermediate and medium energies is presented. The main results on space-time access to reaction dynamics are discussed with a look at future perspectives to obtain probes of the density dependence of the symmetry energy.
\end{abstract}

Particle-particle correlations offer tools to study space-time properties in reaction dynamics [1] and certain spectroscopic information about unbound states $[2,3]$. On one hand the sensitivity to space-time properties of the reaction has led to an extensive use of intensity interferometry in nuclear reactions at both intermediate and relativistic energies $[1,4]$. On the other hand, if two or more particles can be detected as decay products of a specific unbound state produced during the reaction the measured correlation functions contain spectroscopic information about their parent unbound state. This has recently led to use correlations also as a tool for spectroscopy $[2,3,5]$. In this respect one can reasonably state that heavy-ion collisions and multi-particle correlations represent a rich laboratory to study nuclear systems.

\section{Femtoscopy in heavy-ion collisions}

We nowadays know how to explore the spatial extent and the lifetime of particle emitting sources produced in heavy-ion collisions by using intensity

This is an Open Access article distributed under the terms of the Creative Commons Attribution License 2.0, which permits unrestricted use, distribution, and reproduction in any medium, provided the original work is properly cited. 
interferometry $[6,7]$. The measurement of correlations at small relative momentum leads us to measure distances as small as $10^{-15} \mathrm{~m}$ and time intervals as short as $10^{-23}-10^{-20} \mathrm{~s}$. This is an important achievement, but it does not necessarily correspond always to positive advances in understanding reaction dynamics. Indeed, the emission of particles in heavy-ion collisions can be attributed to several sources, ranging from pre-equilibrium emission to secondary decay evaporation. This makes the interpretation of correlation functions difficult, as it has been extensively discussed in the literature of two-proton correlation functions $[1,8]$. Early studies on inclusive measurements [9] need nowadays more exclusive investigations by covering a large portion of the solid angle with enough granularity and angular resolution. Successful attempts to measure two-proton correlation functions with specific gates on impact parameter, reaction plane and other global variables in a $4 \pi$ detector setup can be found at both intermediate energies $(\mathrm{E} / \mathrm{A}=50$ $100 \mathrm{MeV})[10,11]$ and medium energies (E/A=100-1000 MeV) $[12-14,16,43]$. In the case of intermediate energies central collisions are commonly selected by means of charged particle multiplicity or total transverse energy (see for example the recent work [11]). At medium energies the decay of target spectator participants produced in $\mathrm{Au}+\mathrm{Au}$ at $\mathrm{E} / \mathrm{A}=1000 \mathrm{MeV}$ has been investigated by the ALADiN collaboration in peripheral collisions $[12,13]$ by using the detection of projectile spectators as a filter for impact parameter and excitation energy. In Ref. [14] the same apparatus was used to study correlations from central collisions. At even higher energies the FOPI collaboration has nicely investigated several effects of two-particle correlations by selecting both impact parameter and reaction plane $[16,43]$. These studies, especially in central collisions, have also provided important information on the role played by collective motion. The possibility of learning about emission time-scales of complex fragments requires $4 \pi$ detectors such as Indra and Chimera coupled to high resolution devices with position sensitive detectors and especially designed for correlation measurements. These are generally most suited to study light particle correlations.

\section{Proton femtoscopy}

Protons and the most extensively used particle in HBT studies. The shape of the two-proton correlation function is dominated by the nuclear and Coulomb final state interaction and by their quantum statistics [8]. The anstisymmetrization of the two-body wave function makes the correlation function sensitive to the emission time delay between the two coincident pro- 
tons. This is formally stated by the so-called Koonin-Pratt (KP) equation, $1+R_{\vec{P}}(\vec{q})=1+\int S_{\vec{P}}(\vec{r}) \cdot K(\vec{q}, \vec{r}) \cdot d \vec{r}$ that relates the measured two-proton correlation function, $1+R_{\vec{P}}(\vec{q})$ to the two-particle emitting source, $S_{\vec{P}}(\vec{r})$, defined as the probability of emitting two protons with a relative distance $\vec{r}$ measured at the time when the second particle is emitted. In the above expression $\vec{q}$ and $\vec{P}$ are, respectively, the momentum of relative motion and the total momentum of the pair. The kernel function, $K(\vec{r}, \vec{q})$, contains all the information about the final state interaction between the two coincident protons, including their quantum statistics as identical fermions [8]. Since the kernel is well know for protons, resolving the KP-equation consists of extracting the source function from the measured correlation function. Different approaches have been proposed. They can be summarized as:

- Model source approaches where a specific functional (i.e. Gaussian, spherical, etc.) for the shape of the source is assumed $[9,12,17,43]$. In this case the source depends on one free parameters (typically a Gaussian or spherical radius) that is extracted by fitting the KP-equation to the experimental $1+R(q)$.

- Shape-analysis approaches where the KP-equation is numerically inverted to extract the source function profile, $S(r)$, in a less modeldependent context $[17,18]$; no pre-assumptions about the shape of the source are made. Among these approaches imaging represents the most recent and explored one [17].

- Transport model approaches where the measured correlation function is compared to the one calculated within the final phase-space distribution of a heavy-ion collision simulation with transport models. This approach allows one to probe tranport properties of nuclear matter such as nucleon-nucleon collisions cross sections [19] and the density dependence of the symmetry energy [20].

By using Gaussian model sources, both the spatial extent and the emission lifetime can be disentangled by means of directional correlations. These techniques consist of studying the correlation functions obtained by applying gates on the relative angle between momentum of relative motion, $\vec{q}$, and total momentum, $\vec{P}[21,24]$. The sensitivity of directional correlations to space and lifetime is due to the quantum statistics nature of the two-proton system [8].

One of the first and most successful studies of proton emission lifetimes was performed at intermediate energies [21]. Directional two-proton correlations measured in central $\mathrm{Ar}+\mathrm{Sc}$ collisions at $\mathrm{E} / \mathrm{A}=80 \mathrm{MeV}$ were analyzed 

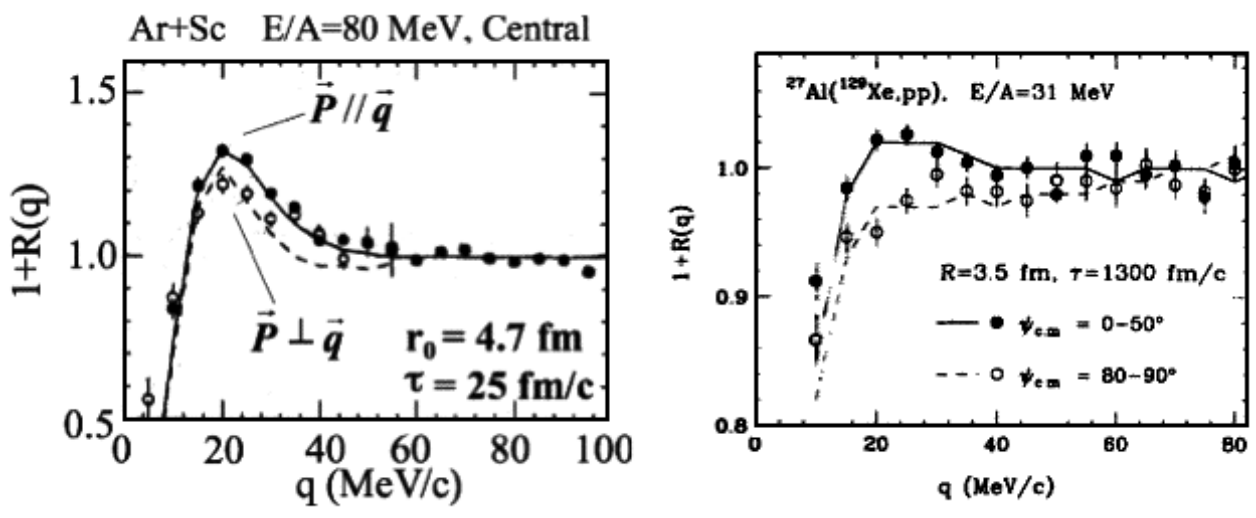

Figure 1: Two-proton directional correlations in central $\mathrm{Ar}+\mathrm{Sc}$ at $\mathrm{E} / \mathrm{A}=80 \mathrm{MeV}$ (left panel) and in evaporation residues produced in inverse kinematics in $\mathrm{Xe}+\mathrm{Al}$ collisions at $\mathrm{E} / \mathrm{A}=31 \mathrm{MeV}$ (right panel, adapted from [21] and [24].)

with gaussian emitting sources providing radii or the order of $4.7 \mathrm{fm}$ and lifetimes of the order of $25 \mathrm{fm} / \mathrm{c}$ (see Fig.1, left panel). Similar values for proton emission lifetimes were extracted also at relativistic energies by studying the decay of target spectators produced in $\mathrm{Au}+\mathrm{Au}$ collisions at $\mathrm{E} / \mathrm{A}=1000$ $\mathrm{MeV}$ [12]. It is important to stress the fact that detector thresholds can be important for a correct interpretation of the obtained results. Indeed, if low energy protons cannot be measured, then evaporative emission are hardly probed by experimental data. This results in an apparent reduction of emission timescales. Attempts to measure longer lifetimes were made in Ref. [22] where $\mathrm{S}+\mathrm{Ag}$ at $\mathrm{E} / \mathrm{A}=22.3 \mathrm{MeV}$ were studied, and in Ref. [23] where proton lifetime effects were searched for in $\mathrm{Ar}+\mathrm{Ag}$ at $\mathrm{E} / \mathrm{A}=44 \mathrm{MeV}$. The results are not easy to be interpreted. More quantitative results can be found in Ref. [24] where lifetime effects have been searched for in inverse kinematics measurements of $\mathrm{Xe}+\mathrm{Al}$ collisions at $\mathrm{E} / \mathrm{A}=31 \mathrm{MeV}$ (see right panel on Fig.1). This study has provided proton emission lifetimes of $\tau \approx$ $1300 \mathrm{fm} / \mathrm{c}$, consistent with a more evaporative scenario as compared to previous results. On the other hand, participant matter at medium energies has been found to be characterized by very short lifetimes. This has been discussed by the FOPI collaboration in studying central $\mathrm{Au}+\mathrm{Au}$ collisions at $\mathrm{E} / \mathrm{A}=400 \mathrm{MeV}$ [43]. The obtained short emission lifetimes are consistent with a violent and almost simultaneous proton emission process by highly excited nuclear system. 


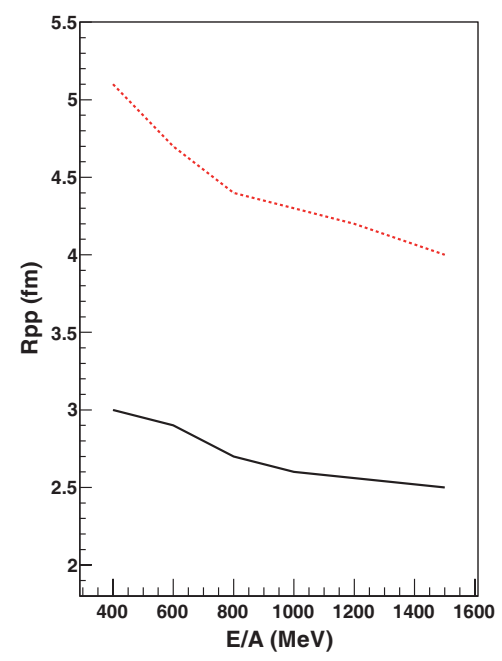

Figure 2: Two-proton source size measured in central $\mathrm{Au}+\mathrm{Au}$ (upper dashed line) and $\mathrm{Ca}+\mathrm{Ca}$ (lower solid line) collisions as a function of the beam energy. [16].)

In Ref. [16] a study of two-proton source size in central $\mathrm{Ca}+\mathrm{Ca}$ and $\mathrm{Au}+\mathrm{Au}$ and as a function on the incident energy $(\mathrm{E} / \mathrm{A}=400-1500)$ provides very nice results in terms of how the emission volume changes with the violence of the collision. The extracted two-proton gaussian radii, as reported on Ref. [16] are plotted on Fig. 2 as a function of the incident energy. The upper dashed line and bottom solid line correspond, respectively to central $\mathrm{Au}+\mathrm{Au}$ and $\mathrm{Ca}+\mathrm{Ca}$ collisions. As expected in a purely geometric size scenario, proton radii are smaller for the $\mathrm{Ca}+\mathrm{Ca}$ system as compared to the $\mathrm{Au}+\mathrm{Au}$ system. The observed decrease of two-proton source sizes with incident energy seems to be consistent with an increasingly large the density of the emitting source. In these reactions a special role is played by the strong collective motion that is built up in participant decay. Collective motion induce position-momentum correlations that result in a reduction of the apparent source size $[10,16,43]$. This effect must be taken into account when studying central collisions at medium energies.

The Aladin collaboration has studied two-proton and other light particle correlations in target spectators produced in $\mathrm{Au}+\mathrm{Au}$ reactions at $\mathrm{E} / \mathrm{A}=1000$ $\mathrm{MeV}[12,13]$. The impact parameter and the deposited spectator excitatin energy has been used as a parameter to isolate sources. The obtained results 
suggest a moderate expansion towards densities lower than saturation, in agreement with the assumptions of statistical multifragmentation models. The statistical scenario is not confirmed by directional correlation studies due to the relatively high detection thresholds [12].

Two-proton correlation function are also sensitive to relative contributions between fast pre-equilibrium sources, and slow sources evaporating protons over longer time-scales [17]. This sensitivity and imaging analysis techniques provide also tools to compare emitting source profiles to transport model simulations $[19,20]$. Comparisons to transport models have also been studied at higher energies [16], where less complex fragments are produced and contributions from evaporative emissions are expected to be strongly reduced.

Light complex particles are characterized by a complex structure due to the presence of resonances induced by nuclear final state interactions $[27,28]$ (see also [1] and Refs. therein). The study of all particle species in the same experiment is expected to provide more clear information about the overall space-time properties of light particle emission in heavy-ion collisions. Reviews of such comparative measurements can be found in $[1,9]$. The available results seem to indicate emitting source sizes that do not scale with the mass of the particles. Smaller d- $\alpha$ radii are reported in Ref. [9]. Besides the fact that different particles may originate from different stages and sources produced during the reaction, the effects induced by collective motion (expansion, rotation on the reaction plane, etc.) must also be considered as a possible distorting effect on correlation functions [10,29]. As a consequence, a reliable comparative study of multiple two-particle correlations requires using a highly performing $4 \pi$ detector array that allows one to characterize correctly the collision event (impact parameter, collective motion, reaction plane). The systematic study of reaction plane and impact parameter effect performed by the FOPI collaboration in Ref. [29] is presently one of the most comprehensive ones. Similar studies need to be addressed at intermediate energies.

\section{MF-IMF correlations}

IMF-IMF correlations allow one to extract complex fragment emission times. This is important for a more clear understanding of multifragmentation mechanisms. The shape of IMF-IMF correlation functions is mostly dominated by Coulomb repulsive final state interactions ( [1] and Refs. therein). Multi-body Coulomb trajectory models has provided IMF emission times 
that decrease with the velocity of the fragments [30,31], indicating that longer IMF emission times can occur when the system is more thermalized. Decreasing emission times are also observed as one increases the beam energy in central collisions [32], suggesting more violent and faster fragment emission in central collisions between $\mathrm{E} / \mathrm{A}=35 \mathrm{MeV}$ and $55 \mathrm{MeV}$.

IMF-IMF correlations have also been used to study the possible dynamical signals of phase transitions in peripheral asymmetric reactions at relativistic energies [33], to disentangle between slow surface evaporations and fast bulk fragment emissions. In the first case, the process is associated with long emission times of the order of $10^{-20}-10^{-21}$ sec, necessary for shape deformation. In contrast, if multifragmentation corresponds to a simultaneous breakup of nuclear matter undergoing a phase transition, the system is expected to fall apart over shorter times $\left(10^{-22}-10^{-23} \mathrm{sec}\right)$, comparable to the timescales involved in the growth of density fluctuations in the spinodal instability region of the nuclear phase diagram.

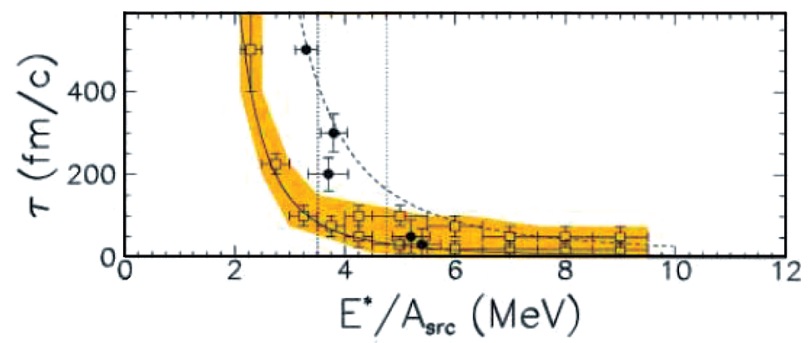

Figure 3: IMF emission lifetimes as a function of the deposited excitation energy measured in target spectators decay induced by the bombardment $\pi^{-}$and $p$ beams at incident momentum of 8.0 and $10.2 \mathrm{GeV} / \mathrm{c}$ (adapted from [33].)

Fig. 3 shows the evolution of IMF emission times with the excitation energy per nucleon measured in $\pi^{-}, \bar{p}+\mathrm{Au}$ at $8.0,8.2,9.2$ and $10.2 \mathrm{GeV} / \mathrm{c}$ with the ISIS array [33]. Extracted emission lifetimes decrease from $\tau \approx 500$ $\mathrm{fm} / \mathrm{c}$ at excitation energies $\mathrm{E}^{*} / \mathrm{A} 2.5 \mathrm{MeV}$ to a saturating value of about $\tau \approx 20-50 \mathrm{fm} / \mathrm{c}$ for excitation energies above $5 \mathrm{MeV} /$ nucleon. These results indicate a transition from a surface evaporation-like emission at low excitation energies towards a bulk simultaneous multifragmentation scenario above excitation energies of the order of $\mathrm{E}^{*} / \mathrm{A}=5 \mathrm{MeV}$ [33].

IMF-IMF correlations at intermediate energies have also been used recently to probe the shape of the of the multifragmenting source with the Indra detector array [34] and to measure neck emission timescales in semiperipheral collisions with the Chimera multi-detector. The literature on 
IMF-IMF correlations in central collisions shows that sub-saturation densities $\left(\rho \approx 0.1-0.6 \rho_{0}\right)$ have been probed, with indications of transitions from sequential slow emissions at low excitation to fast emissions when higher excitation and more explosive scenarios are reached. Due to the sensitivity to collective motion, it will be important to improve our measurements of IMF-IMF correlations by means of $4 \pi$ detector arrays coupled to high resolution correlators. A different application of correlation measurements is shown on Ref. [36] where IMF-Light Particle correlations are used by the Indra collaboration to characterize the excitation energy and mass/charge ratios of primary fragments before they undergo secondary decays.

\section{Correlations as spectroscopy tools}

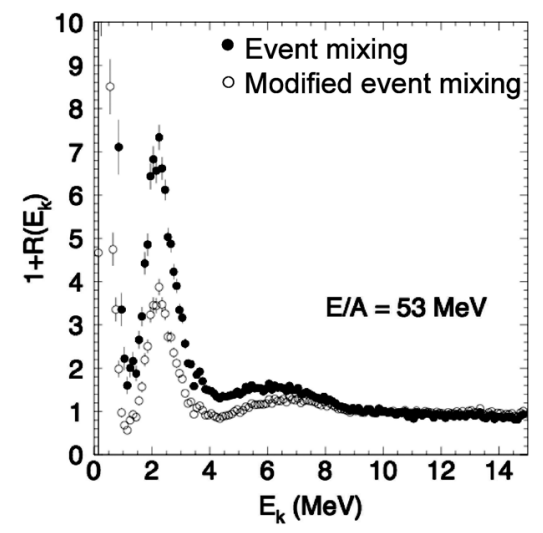

Figure 4: Three-alpha correlation functions in $\mathrm{C}+\mathrm{Mg}$ reactions at $\mathrm{E} / \mathrm{A}=53 \mathrm{MeV}$ (filled dots). The open dots are obtained with a modified definition of the event mixing backgrouond (see text and Ref. [5] for details).

Heavy-ion collisions can also be used to explore the spectroscopic properties of unbound states abundantly produced during the evolution of the system. These unstable states can be identified and explored by detecting all the products of their decay in coincidence with an experimental setup characterized by a high angular and energy resolution. In Ref. [2] p- ${ }^{7} \mathrm{Be}$ correlation functions have been measured in $\mathrm{Xe}+\mathrm{Au}$ at $\mathrm{E} / \mathrm{A}=50 \mathrm{MeV}$ central collisions to probe the spin of internal states in ${ }^{8} \mathrm{~B}$ nuclei. More recently analyses of two-, three- and four-particle correlation functions have provided access to the decay properties of highly lying unbound states in ${ }^{12} \mathrm{C}$ and ${ }^{10} \mathrm{C}$ nuclei [5]. These nuclei were produced as excited projectile-like fragments 
$\left(\mathrm{PLF}^{*}\right)$ in ${ }^{12} \mathrm{C}+{ }^{24} \mathrm{Mg}$ peripheral collisions at $\mathrm{E} / \mathrm{A}=53$ and $95 \mathrm{MeV}$ [5]. As an exmple Fig. 4 shows the three- $\alpha$ correlation function and the peaks correspond to ${ }^{12} \mathrm{C}$ internal states. Comparing the correlation function with the one obtained using different event mixing techniques one can qualitatively disentangle direct three-body decay and sequential emission mechanisms proceeding through the formation of unbound ${ }^{8}$ Be state. Similar procedures allow one to estimate the relative contributions between simultaneous decays of ${ }^{10} \mathrm{C}^{*}$ nuclei into two protons and two alphas and sequential processes producing intermediate states in ${ }^{6} \mathrm{Be},{ }^{8} \mathrm{Be}$ and ${ }^{9} \mathrm{~B}[5]$. These results have to be taken as exploratory studies: higher resolution measurements are indeed required in order to perform more quantitative analyses of such unbound states (see [37-39]).

\section{Intensity interferometry and perspectives in symmetry energy research}

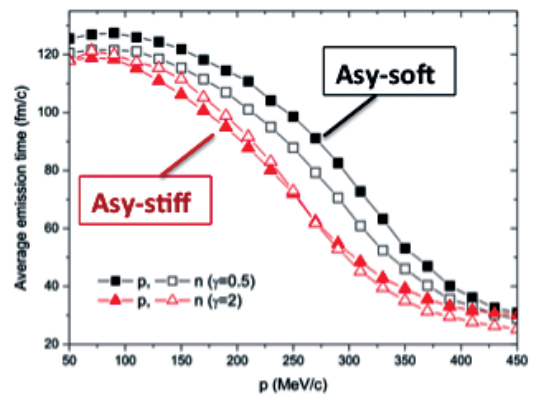

Figure 5: Predicted average emission times of neutrons (open data points) and protons (solid data points) as a function of their momentum in IBUU simulations of ${ }^{52} \mathrm{Ca}+{ }^{48} \mathrm{Ca}$ central collisions at $\mathrm{E} / \mathrm{A}=80 \mathrm{MeV}$ (adapted from [20].

Two-nucleon (nn, np, and $\mathrm{pp}$ ) correlations are expected to be sensitive to the density dependence of the symmetry energy [1,20], but little experimental information is still available. Fig. 5 shows the predicted average emission times of neutrons (open data points) and protons (solid data

points) as a function of their momentum in IBUU simulations of ${ }^{52} \mathrm{Ca}+{ }^{48} \mathrm{Ca}$ central collisions at $\mathrm{E} / \mathrm{A}=80 \mathrm{MeV}$. The black (red) symbols refer simulations performed with a softer (stiffer) density dependence of the symmetry energy, defined in terms of the exponent parameter $\gamma[20]$. These simulations suggest that the relative neutron-proton emission time at medium momenta (around $p \approx 250 \mathrm{MeV} / \mathrm{c}$ ) depend on the symmetry energy at subsaturation. 
Emission chronology for neutrons and protons is therefore expected to provide information about the EoS for asymmetric nuclear matter. Attempt to probe chronology have been presented in Ref. [41]. The technique is based on measuring neutron-proton correlation functions following the ideas proposed by Lednicki et al., in Ref. [42]. Velocity gated neutron-proton correlations are indeed capable of probing catch-up effects in final state interactions: if a proton is emitted after a neutron but with a larger velocity, then it will have a higher chance of catching it in its flight path, as compared to the case when the neutron is emitted earlier (see [42] for more complete explanation of this technique).

Therefore, comparing n-p correlation functions with $v_{p}<v_{n}$ and with $v_{n}<v_{p}$ is expected to provide an observable that should suggest which of the two particle was emitted first $[41,42]$. Similar studies have been successfully performed by the FOPI collaboration [43], the Indra collaboration [44] and the Chic collaboration [41]. Earlier studies can be found also in Ref. [45]. They represent an important perspective for the future both at intermediate energies and at medium energies.

\section{Conclusions}

In this contribution a review of some of the important results and perspectives offered by particle-particle correlations is presented. Correlations between light particles have been extensively explored and have provided important information about proton emission times and the radius of their emitting sources. Intermediate mass fragment correlations have also provided important results in terms of multifragmentation times, allowing one to disentangle simultaneous vs sequential emissions. Similar correlation chronology analyses are a key for exploring the details of nuclear dynamics and are expected to provide important information also for the study of decay modes in unstable states of exotic nuclei. The work done by our community can be considered successful due to the ability of measuring sizes in femtoscopic systems. On the other hand more work is still required to better understand low energy particle HBT (both theoretically and experimentally) and to clarify the observed differences in radii extracted from different particle species. This asks for complete-event experiments using $4 \pi$ detectors and high resolution arrays that can address the rich information contained in multi-particle correlation measurements. 


\section{References}

[1] G. Verde et al, Eur. Phys. J. A 30, 81 (2006).

[2] W.P. Tan et al., Phys. Rev. C69, 061304 (2004).

[3] R.J. Charity et al., Phys. Rev. C80, 024306 (2009)

[4] M. A. Lisa et al., Ann. Rev. Nucl. Part. Sci. 55, 357 (2005).

[5] F. Grenier et al., Nucl. Phys. A811, 233 (2008)

[6] R. Hanbury-Brown and R.Q. Twiss, Nature 178, 1046 (1956).

[7] G. Goldhaber et al., Phys. Rev. Lett. 3, 181 (1959); Phys. Rev. 120, 300 (1960).

[8] S. E. Koonin, Phys. Lett. B 70, 43 (1977).

[9] D.H. Boal, C.K. Gelbke, B.K. Jennings, Rev. Mod. Phys. 62, 553 (1990).

[10] G. Verde et al., Phys. Lett. B653, 12 (2007).

[11] V. Henzl et al., Phys. Rev. C85, 014606 (2012).

[12] C. Schwarz et al., Nucl. Phys. A 681, 279 (2001).

[13] S. Fritz et al., Phys. Lett. B461, 315 (1999).

[14] V. Serfling et al., Phys. Rev. Lett. 80, 3928 (1998).

[15] R. Kotte et al., Eur. Phys. J. A6, 185 (1999).

[16] R. Kotte et al., Eur. Phys. J. A23, 271 (2005).

[17] G. Verde et al., Phys. Rev. C65, 054609 (2002).

[18] D.A. Brown et al., Phys. Rev. C64, 014902 (2001).

[19] G. Verde et al., Phys. Rev. C67, 034606 (2003).

[20] L.W. Chen et al., Phys. Rev. C68, 014605 (2004).

[21] M. A. Lisa et al., Phys. Rev. Lett. 71, 2863 (1993).

[22] T. C. Awes et al., Phys. Rev. Lett. 61, 2665 (1988). 
[23] D. Goujdami et al., Z. Phys. A339, 293 (1991).

[24] M.A. Lisa et al., Phys. Rev. C49, 2788 (1994).

[25] M. A. Lisa et al., Phys. Rev. Lett. 70, 3709 (1993).

[26] W. G. Gong et al., Phys. Rev. C43, 1804 (1991).

[27] J. Pochodzalla et al., Phys. Rev. C35, 1695 (1987).

[28] T.K. Nayak et al., Phys. Rev. C45, 132 (1992).

[29] R. Kotte et al., Phys. Rev. C51, 2686 (1995).

[30] T. Glasmacher et al., Phys. Rev. C50, 952 (1994)

[31] E. Cornell et al., Phys. Rev. Lett. 75, 1475 (1995).

[32] E. Bauge et al., Phys. Rev. Lett. 70, 3705 (1993).

[33] L. Beaulieu et al., Phys. Rev. Lett. 84, 5791 (2000).

[34] A. Le Fevre et al., Phys. Lett. B659, 807 (2008).

[35] E. De Filippo et al, 71, 044602 (2005).

[36] N. Marie et al., Phys. Rev. C58, 256 (1998); S. Hudan et al., Phys. Rev. C67, 064613 (2003).

[37] R.J. Charity et al., Phys. Rev. C75, 051304 (2007).

[38] N. Curtis et al., Phys. Rev. C 77, 021301 (2008).

[39] R.J. Charity et al., PRC76, 064313 (2007); R.J. Charity et al., PRC78, 054307 (2008).

[40] R. Ghetti et al., Phys. Rev. C70, 034601 (2004).

[41] R. Ghetti et al, Phys. Rev. Lett. 91, 092701 (2003).

[42] R. Lednicky et al., Phys. Lett. B373, 30 (1996).

[43] R. Kotte et al., Eur. Phys. J. A6, 185 (1999).

[44] D. Gourio et al., Eur. Phys. J. A7, 245 (2000).

[45] C.J. Gelderloos et al., Phys. Rev. Lett. 75, 3082 (1995); C.J. Gerderloos and J.M. Alexander, Nucl. Instr. Meth. A349, 618 (1994). 